University of Texas at El Paso

ScholarWorks@UTEP

$9-2003$

\title{
A Full Function-Based Calculus of Directed and Undirected Intervals: Markov's Interval Arithmetic Revisited
}

Juergen Wolff von Gudenberg

Vladik Kreinovich

The University of Texas at El Paso, vladik@utep.edu

Follow this and additional works at: https://scholarworks.utep.edu/cs_techrep

Part of the Computer Engineering Commons

Comments:

Technical Report: UTEP-CS-03-01a

Published in Numerical Algorithms, 2004, Vol. 37, pp. 417-428.

\section{Recommended Citation}

von Gudenberg, Juergen Wolff and Kreinovich, Vladik, "A Full Function-Based Calculus of Directed and Undirected Intervals: Markov's Interval Arithmetic Revisited" (2003). Departmental Technical Reports (CS). 275.

https://scholarworks.utep.edu/cs_techrep/275

This Article is brought to you for free and open access by the Computer Science at ScholarWorks@UTEP. It has been accepted for inclusion in Departmental Technical Reports (CS) by an authorized administrator of ScholarWorks@UTEP. For more information, please contact Iweber@utep.edu. 


\title{
A Full Function-Based Calculus of Directed and Undirected Intervals: Markov's Interval Arithmetic Revisited
}

\author{
Jürgen Wolff von Gudenberg ${ }^{1,2}$ \\ and Vladik Kreinovich ${ }^{2}$ \\ ${ }^{1}$ Lehrstuhl für Informatik II \\ Universität Würzburg \\ Am Hubland \\ D-97074 Würzburg, Germany \\ email wolff@informatik.uni-wuerzburg.de \\ ${ }^{2}$ Department of Computer Science \\ University of Texas at El Paso \\ $500 \mathrm{~W}$. University \\ El Paso, TX 79968, USA \\ email vladik@cs.utep.edu
}

\begin{abstract}
This paper proposes a new interpretation of intervals as classes of functions having the same domain. Interval operations are seen as operations on these classes. This approach allows to recover Markov's directed interval arithmetic by taking into account the monotonicity of the functions.
\end{abstract}

Keywords: Interval computations; functional interpretation

AMS Subject Classification: 65G20, 65G30, 65G40

\section{Introduction: Why Function-Based Calculus of Intervals}

This paper proposes a new interpretation of intervals as classes of functions having the same domain. Interval operations are seen as operations on these classes. This approach allows to recover Markov's directed interval arithmetic by taking into account the monotonicity of the functions.

Why do we interpret intervals as classes of functions? One of the main objectives of interval computations is $[1,6,7,14]$ :

Given: intervals $\left[\underline{x}_{1}, \bar{x}_{1}\right], \ldots,\left[\underline{x}_{n}, \bar{x}_{n}\right]$, and a continuous function $f\left(x_{1}, \ldots, x_{n}\right)$, Compute: the range

$\left.[\underline{y}, \bar{y}] \stackrel{\text { def }}{=} f\left(\left[\underline{x}_{1}, \bar{x}_{1}\right], \ldots, \underline{x}_{n}, \bar{x}_{n}\right]\right)=\left\{f\left(x_{1}, \ldots, x_{n}\right) \mid x_{1} \in\left[\underline{x}_{1}, \bar{x}_{1}\right] \& \ldots \& x_{n} \in\left[\underline{x}_{n}, \bar{x}_{n}\right]\right\}$

of the given function $f$ over the given intervals $\left[\underline{x}_{i}, \bar{x}_{i}\right]$. 
From this viewpoint, the resulting interval $[\underline{y}, \bar{y}]$ means that we have a function whose range is contained in this interval. In other words, it seems reasonable to associate each interval $\mathbf{y}=[\underline{y}, \bar{y}]$ with the set of all continuous functions whose range is equal to $\mathbf{y}$.

A problem with this definition is that computing the exact range is difficult; the problem of computing the range is, in general, NP-hard (see, e.g., [8]). As a result, in practice, our main objective is not necessarily to compute the exact range, but rather to compute an enclosure $[\underline{Y}, \bar{Y}] \supseteq$ $[\underline{y}, \bar{y}]$ for the desired range $[y, \bar{y}]$ (and to make this enclosure as narrow as possible).

From this practical viewpoint, we would rather associate each interval $\mathbf{Y}=[\underline{Y}, \bar{Y}]$ with the set of all continuous functions whose range is a subset of $\mathbf{Y}$.

The simplest case is when we consider functions $f(x)$ of one variable $x=x_{1}$, with $\left[\underline{x}_{1}, \bar{x}_{1}\right]=[0,1]$. Then, the range is $f([0,1])$, and each interval $[\underline{y}, \bar{y}]$ is associated with the class $F$ of all continuous functions $f:[0,1] \rightarrow R$ for which $f([0,1]) \subseteq[\underline{y}, \bar{y}]$.

For every arithmetic operation $\odot(+, \cdot \cdot$, etc. $)$, we can define, for every two functions $f(x)$ and $g(x)$, the result $f \odot g$ as a function for which, for every $x,(f \odot g)(x)=f(x) \odot g(x)$, and then for

every two classes $F$ and $G$, we can define $F \odot G \stackrel{\text { def }}{=}\{f \odot g \mid f \in F \& g \in G\}$. We will show that this definition leads to standard interval arithmetic.

S. Markov showed (see, e.g., $[11,12]$ ) that if we consider classes of monotonic functions, we get Kaucher arithmetic (originally proposed in $[2,3,4,5,15]$ ). This is a very interesting result because Kaucher arithmetic has many applications: e.g., it is useful in solving systems of interval linear equations $[9,13,16,17]$, and in practical problems related to interpolation [13], to system identification under uncertainty [16, 17], and to control [16].

In this paper, we propose a general definition of functional intervals, describe explicit expressions for arithmetic operations with such "intervals", and show that these expressions lead to Markov's directed intervals and Kaucher arithmetic.

\section{Describing This Idea in Formal Terms}

\subsection{Simplest Case}

The simplest case of the above problem of interval computations is when we consider functions $f(x)$ of only one variable $x=x_{1}$, and we take the simplest possible interval $\left[\underline{x}_{1}, \bar{x}_{1}\right]=[0,1]$.

\subsection{Resulting Definition}

In this case, the range is $f([0,1])$, and each interval $[\underline{a}, \bar{a}]$ is associated with the class of all continuous functions $f:[0,1] \rightarrow R$ for which $f([0,1]) \subseteq[\underline{a}, \bar{a}]$. In other words, we arrive at the following definition:

$$
[\underline{a}, \bar{a}]_{F} \stackrel{\text { def }}{=}\{f \mid f \in C \& f([0,1]) \subseteq[\underline{a}, \bar{a}]\},
$$

where $C$ denotes the class of all continuous functions.

\subsection{Operations on Intervals}

Standard operations of interval arithmetic can be naturally interpreted in this functional form. Indeed, for every arithmetic operation $\odot(+,-, \cdot$, etc. $)$, we can define,

- for every two functions $f(x)$ and $g(x)$, the result $f \odot g$ as a function for which, for every $x$, $(f \odot g)(x)=f(x) \odot g(x)$ (i.e., a component-wise operation); and then 
- for every two classes $F$ and $G$, we can define

$$
F \odot G=\{f \odot g \mid f \in F \& g \in G\} .
$$

How are these operations related to standard operations on intervals? It turns out that this definition leads to standard interval arithmetic, for example:

Theorem 1. $[\underline{a}, \bar{a}]_{F}+[\underline{b}, \bar{b}]_{F}=[\underline{a}+\underline{b}, \bar{a}+\bar{b}]_{F}$.

Proof. To prove the equality of the two sets, we must prove that every element from the left set belongs to the right set, and vice versa.

$1^{\circ}$. Let us first prove that every element $h(x)$ of the set $[\underline{a}, \bar{a}]_{F}+[\underline{b}, \bar{b}]_{F}$ belongs to the set $[\underline{a}+\underline{b}, \bar{a}+\bar{b}]_{F}$.

Indeed, let $h \in[\underline{a}, \bar{a}]_{F}+[\underline{b}, \bar{b}]_{F}$. By definition of the sum of two function classes, the fact that the function $h(x)$ belongs to the sum of the two function classes means that $h(x)$ can be represented as a (component-wise) sum $h(x)=f(x)+g(x)$ of two function $f(x)$ and $g(x)$, where $f \in[\underline{a}, \bar{a}]_{F}$ and $g \in[\underline{b}, \bar{b}]_{F}$.

By definition of these classes, $f \in[\underline{a}, \bar{a}]_{F}$ means that $f([0,1]) \subseteq[\underline{a}, \bar{a}]$, i.e., that for every $x \in[0,1]$, we have

$$
\underline{a} \leq f(x) \leq \bar{a} .
$$

Similarly, we conclude that for every $x \in[0,1]$, we have

$$
\underline{b} \leq g(x) \leq \bar{b} .
$$

Adding the two displayed inequalities, we conclude that for every $x \in[0,1]$, we have

$$
\underline{a}+\underline{b} \leq h(x)=f(x)+g(x) \leq \bar{a}+\bar{b} .
$$

In other words, we have $h([0,1]) \subseteq[\underline{a}+\underline{b}, \bar{a}+\bar{b}]$. By definition of the class $[., .]_{F}$, this means that $h \in[\underline{a}+\underline{b}, \bar{a}+\bar{b}]_{F}$.

$2^{\circ}$. To complete our proof, let us show that every element $h \in[\underline{a}+\underline{b}, \bar{a}+\bar{b}]_{F}$ also belongs to the set $[\underline{a}, \bar{a}]_{F}+[\underline{b}, \bar{b}]_{F}$

Indeed, let $h \in[\underline{a}+\underline{b}, \bar{a}+\bar{b}]_{F}$. To prove this statement, we must be able to represent the function $h(x)$ in the form $f(x)+g(x)$, where $f \in[\underline{a}, \bar{a}]_{F}$ and $g \in[\underline{b}, \bar{b}]_{F}$. For that, we will define an auxiliary function $F:[\underline{a}+\underline{b}, \bar{a}+\bar{b}] \rightarrow[\underline{a}, \bar{a}]_{F}$ and then take $f(x) \stackrel{\text { def }}{=} F(h(x))$ and $g(x) \stackrel{\text { def }}{=}$ $h(x)-F(h(x))$. We want to define the function $F(z)$ in such a way that for every real number $z$ for which this function is defined, we have $\underline{a} \leq F(z) \leq \bar{a}$ (this is true for any mapping to the interval $[\underline{a}, \bar{a}])$ and $\underline{b} \leq z-F(z) \leq \bar{b}$. Then, we will automatically have $f(x) \in[\underline{a}, \bar{b}]$ and $g(x) \in[\underline{b}, \bar{b}]$, hence $f \in[\underline{a}, \bar{a}]_{F}$ and $g \in[\underline{b}, \bar{b}]_{F}$.

We define this function $F(z)$ as follows:

- $F(z)=z-\underline{b}$ for $\underline{a}+\underline{b} \leq z \leq \bar{a}+\underline{b}$, and 
- $F(z)=\bar{a}$ for $\bar{a}+\underline{b} \leq z \leq \bar{a}+\bar{b}$.

In this case,

- when $\underline{a}+\underline{b} \leq z \leq \bar{a}+\underline{b}$, we have $z-F(z)=\underline{b} \in[\underline{b}, \bar{b}]$;

- when $\bar{a}+\underline{b} \leq z \leq \bar{a}+\bar{b}$, then, by subtracting $\bar{a}=F(z)$ from all three terms in this inequality, we conclude that $\underline{b} \leq z-F(z) \leq \bar{b}$.

In both cases, $z-F(z) \in[\underline{b}, \bar{b}]$, so we get the desired function $F(z)$. The theorem is proven.

Comment. Similar results can be proven for other operations of interval arithmetic (e.g., for subtraction, the proof is basically the same as for addition). So, from the viewpoint of interval computations, we can simply identify each interval $[\underline{a}, \bar{a}]$ with the corresponding class of functions $[\underline{a}, \bar{a}]_{F}$.

\section{$3 \quad$ Beyond Standard Intervals}

We have already mentioned that sometimes, we succeed in getting the exact range, but most often, we get an enclosure. It is desirable to separate these two cases, i.e., to consider, in addition to the formula (1), the class of functions for which the range is exactly equal to $[\underline{a}, \bar{a}]$. We will denote this class by

$$
\dot{i}, \bar{a}] \stackrel{\text { def }}{=}\{f \mid f \in C \& f([0,1])=[\underline{a}, \bar{a}]\} .
$$

An important case when we can easily compute the exact range is when a function $f(x)$ is monotonic:

- If we know that the function $f(x)$ is non-decreasing (denoted $f \uparrow$ ), then we can compute the range as $f([0,1])=[f(0), f(1)]$.

- If we know that the function $f(x)$ is non-increasing (denoted $f \downarrow$ ), then we can compute its range as $f([0,1])=[f(1), f(0)]$.

It is therefore reasonable to consider the corresponding sets of monotonic functions:

$$
\begin{aligned}
& {[\underline{a}, \bar{a}] \rightarrow \stackrel{\text { def }}{=}\{f \uparrow \mid f \in C \& f([0,1]) \subseteq[\underline{a}, \bar{a}]\} ;} \\
& {[\underline{a}, \bar{a}] \leftarrow \stackrel{\text { def }}{=}\{f \downarrow \mid f \in C \& f([0,1]) \subseteq[\underline{a}, \bar{a}]\} ;} \\
& \dot{[a}, \bar{a}] \rightarrow \stackrel{\text { def }}{=}\{f \uparrow \mid f \in C \& f([0,1])=[\underline{a}, \bar{a}]\} ; \\
& \dot{[a}, \bar{a}] \leftarrow \stackrel{\text { def }}{=}\{f \downarrow \mid f \in C \& f([0,1])=[\underline{a}, \bar{a}]\} .
\end{aligned}
$$

Crudely speaking, the class $[\underline{a}, \bar{a}] \rightarrow$ describes not only the interval itself, but also the direction in which a function $f(x)$ covers this interval.

We will see that naturally defined operations on these classes of functions lead to directed interval arithmetic as defined by S. Markov (see, e.g., [11, 12]). Thus, these classes can be naturally associated with Markov's directed intervals. 


\section{Beyond Standard Operations}

As we have mentioned, if we know that the function is monotonic, then this information drastically simplifies the problem of computing the range of this function. Sometimes, in addition to (or instead of) knowing that the original functions are monotonic, we also know that some intermediate computational results are monotonic. To capture such situations, in addition to the operations (2), it makes sense to consider new "directed" operations in which we additionally require that the functions are monotonic.

Formally, if $\odot$ be an arithmetic operation, and $F$ and $G$ are function classes, then, in addition to the class $F \odot G$ as defined by the formula (2), we can also define the following classes:

$$
\begin{aligned}
& F \odot \rightarrow \stackrel{\text { def }}{=}\{h \uparrow \mid h \in F \odot G\} ; \\
& F \odot \leftarrow G \stackrel{\text { def }}{=}\{h \downarrow \mid h \in F \odot G\} .
\end{aligned}
$$

It is also reasonable to consider the case when the resulting function $h$ is monotonic, but we do not whether it is non-increasing or non-decreasing, i.e., to consider the class

$$
F \odot \leftrightarrow \text { def }\{h \uparrow \vee h \downarrow \mid h \in F \odot G\}=(F \odot \rightarrow G) \cup(F \odot \leftarrow G) .
$$

In the following sections, we will see that the new definitions provide a new interpretation of "directed" operations between directed intervals - as defined by S. Markov.

Comment. It is worth mentioning that this paper is not simply a new interpretation of Markov's operations: our approach also enables us to extend these operations to new types of intervals.

\section{Technical Comment: What Is the Functional Analogue of In- terval Hull?}

\subsection{Problem}

We started with the traditional interval arithmetic. In our interpretation, the traditional interval arithmetic corresponds to the situation when we consider function classes of type (1) (they are analogues of intervals), and to these classes, we apply arithmetic operations of type (2) (they are analogues of arithmetic operations with intervals). The above Theorem 1 says that in this manner, we indeed get a new interpretation of interval arithmetic: if we restrict ourselves to the function classes $[\underline{a}, \bar{a}]_{F}$ and $[\underline{b}, \bar{b}]_{F}$ of type (1) and apply an arithmetic operation $\odot$ to these classes - as defined by the formula (2) - then the result is again a class of type (1), namely, the class $[\underline{c}, \bar{c}]_{F}$, where the corresponding interval

$$
[\underline{c}, \bar{c}]=[\underline{a}, \bar{a}] \odot[\underline{b}, \bar{b}]
$$

is exactly the result of applying the corresponding interval operation $\odot$ to the input intervals $[\underline{a}, \bar{a}]$ and $[\underline{b}, \bar{b}]$.

After that, we have expanded both the objects and the operations. Specifically, instead of simply considering classes of type (1), we now consider classes of types (1), (3)-(7); instead of considering only operations of type (2), we now also consider operations of types (8)-(10).

A natural question is: if we apply one of the new operations to one of the new classes, will we still get a class of one of the known types (1), (3)-(7)? It turns out that the answer to this natural question is negative: namely, the class $A \stackrel{\text { def }}{=}[0,1]+\leftrightarrow[0,1]$ is not equal to any of the classes (1), (3)-(7). 
Indeed, by definition of the operation ${ }^{\leftrightarrow} \leftrightarrow$ (formula (10)), the class $A$ consists of all monotonic (non-increasing or non-decreasing) functions with a range in $[0,1]$. One can see that this class is indeed different from any of the classes (1), (3)-(7).

\subsection{Similar Problem}

A similar problem occurs when we add additional functions to the list of operations of interval arithmetic. Specifically, for arithmetic operations $a \odot b$, the corresponding interval operation $[\underline{a}, \bar{a}] \odot$ $[\underline{b}, \bar{b}]$ can be defined as the range

$$
[\underline{a}, \bar{a}] \odot[\underline{b}, \bar{b}]=\{a \odot b \mid a \in[\underline{a}, \bar{a}] \& b \in[\underline{b}, \bar{b}]\}
$$

of the arithmetic operation $\odot$ on the intervals $[\underline{a}, \bar{a}]$ and $[\underline{b}, \bar{b}]$. Therefore, if we have a function $f\left(x_{1}, \ldots, x_{n}\right)$ from ( $n$-tuples of) real numbers to real numbers, we can define the interval analogue of this function as the range of this function $f$ on the corresponding intervals:

$$
f\left(\left[\underline{x}_{1}, \bar{x}_{1}\right], \ldots,\left[\underline{x}_{n}, \bar{x}_{n}\right]\right) \stackrel{\text { def }}{=}\left\{f\left(x_{1}, \ldots, x_{n}\right) \mid x_{1} \in\left[\underline{x}_{1}, \bar{x}_{1}\right] \& \ldots \& x_{n} \in\left[\underline{x}_{n}, \bar{x}_{n}\right]\right\} .
$$

For continuous functions, this works well, because the range of a continuous function $f\left(x_{1}, \ldots, x_{n}\right)$ on a box $\left[\underline{x}_{1}, \bar{x}_{1}\right] \times \ldots \times\left[\underline{x}_{n}, \bar{x}_{n}\right]$ is always an interval.

However, if we consider discontinuous functions, e.g., the sign function $\operatorname{sign}(x)$ defined as 1 for $x>0,-1$ for $x<0$ and 0 for $x=0$, then its range is not necessarily an interval. For example, $X \stackrel{\text { def }}{=} \operatorname{sign}([-1,1])=\{-1,0,1\}$.

How is this problem similar to the problem described in the previous subsection? In both cases, we started with a class of objects and a class of operations that is closed in the sense that the result of applying each of the given operations to each of the given objects results in the object of the given class. Then, we extended our classes, and we no longer have closeness:

- in the above case, we get a function class that is different from one of the classes (1), (3)-(7);

- in the sign case, we get a range that is different from an interval.

In view of this similarity, in order to solve the above problem, we will recall how the similar problem is solved in traditional interval arithmetic, and extend the main idea behind this solution to our case (of function classes).

\subsection{Usual Solution}

When the range $X$ of a function (like $\operatorname{sign}(x)$ ) is not an interval, and we want to stay within interval arithmetic, we take the narrowest interval enclosure instead of the original range $X$. In other words, instead of the set $X$, we take its interval hull $[X]$ that is defined as the intersection of all the intervals that contain the set $X$ :

$$
[X] \stackrel{\text { def }}{=} \bigcap\{\text { all intervals } \supseteq X\} .
$$

The possibility to define the interval hull as the intersection comes from the fact that the intersection of an arbitrary family of intervals is also an interval (unless, of course, it is empty set).

For example, for the above range $X=\{-1,0,1\}$, the interval hull is equal to $[X]=[-1,1]$. 


\subsection{Natural Idea}

It is natural to define, for functional interval classes, an interval hull of a given set as the intersection of all interval classes which contain this set.

\subsection{Minor Problem}

We are not yet ready to implement this idea because, for this idea to work, we must make sure that the intersection of interval classes is always an interval class, and this is not always the case: the intersection of the class $[\underline{a}, \bar{a}] \rightarrow$ of all non-decreasing functions and the class $[\underline{a}, \bar{a}] \leftarrow$ of all nonincreasing functions is the set of all constant functions, which is neither a directed nor an undirected interval:

$$
[\underline{a}, \bar{a}] \rightarrow \cap[\underline{a}, \bar{a}]^{\leftarrow}=\{f \mid f \in C \& f([0,1]) \subseteq[\underline{a}, \bar{a}] \& f=\text { const }\} .
$$

It is therefore necessary to add such classes to our list of interval classes, i.e., define

$$
[\underline{a}, \bar{a}]=\stackrel{\text { def }}{=}\{f \mid f([0,1]) \subseteq[\underline{a}, \bar{a}] \& f=\text { const }\} .
$$

Comment. At first glance, it may look like we also need to define a class

$$
\dot{[} \underline{a}, \bar{a}]=\stackrel{\text { def }}{=}\{f \mid f([0,1])=[\underline{a}, \bar{a}] \& f=\text { const }\} .
$$

However, thus defined class is non-empty only if the two endpoints $\underline{a}$ and $\bar{a}$ are equal, and the corresponding class (12) is covered by the formula (11).

Let $\mathcal{F}$ denote the family of all classes of the type (1), (3), (4)-(7), and (11). Then, it is easy to prove that the resulting family is closed under intersection:

Theorem 2. The family $\mathcal{F}$ is closed under intersection.

Proof: Main Idea. In order to prove that the intersection belongs to the family, we can first intersect functional intervals belonging to the same type, and then take the intersection of the results.

First, we prove, for each type, that within this type, the intersection belongs to the same family (if it is non-empty at all); this is straightforward.

Then, by enumerating all possible pairs of types, we prove that their intersection is also one of the standard functional intervals; this is somewhat tedious but also straightforward.

Now, we are ready for the final definition:

\subsection{Final Definition}

For each operation $\odot\left(\right.$ such as,$++^{\leftarrow}$, etc. $)$, we define the new operation $\odot \mathcal{F}$ as follows:

$$
F \odot \mathcal{F} G \stackrel{\text { def }}{=} \bigcap\{H \in \mathcal{F} \mid F \odot G \subseteq H\} .
$$

This is the operation that we will be using. 


\section{Example: Markov's Explanation of Kaucher Arithmetic Refor- mulated}

Following Markov, let us consider intervals of the type $[\underline{a}, \bar{a}]^{\leftarrow}$ and $[\underline{a}, \bar{a}] \rightarrow$. To get Kaucher's arithmetic, in this section, we will:

- identify a proper interval $[\underline{a}, \bar{a}](\underline{a} \leq \bar{a})$ with $[\underline{a}, \bar{a}] \rightarrow$, and

- identify an improper interval $[\bar{a}, \underline{a}](\underline{a} \leq \bar{a})$ with $[\underline{a}, \bar{a}] \leftarrow$.

For example, $[0,1]$ will mean $[0,1] \rightarrow$, and $[1,0]$ will mean $[0,1] \leftarrow$. As an operation + , we take $+{ }^{\leftrightarrow} \mathcal{F}$. The following result shows that we do get Kaucher arithmetic (see, e.g., [5]):

Theorem 3. In the above interpretation, for arbitrary real numbers $a_{1}, b_{1}, a_{2}$, and $b_{2}$, we have

$$
\left[a_{1}, b_{1}\right]+\left[a_{2}, b_{2}\right]=\left[a_{1}+a_{2}, b_{1}+b_{2}\right]
$$

Proof. We want to prove that for arbitrary real numbers $a_{1}, b_{1}, a_{2}$, and $b_{2}$, we have

$$
\left[a_{1}, b_{1}\right]+\left[a_{2}, b_{2}\right]=\left[a_{1}+a_{2}, b_{1}+b_{2}\right]
$$

To prove this result, let us recall what an interval $[a, b]$ means in our functional interpretation:

- When $a \leq b$, this interval means the set of all non-decreasing functions $f(x)$ for which the range is exactly $[a, b]$. Since the function is non-decreasing, its smallest value is attained at $x=0$ and its largest value is attained at $x=1$, hence $f(0)=a$ and $f(1)=b$.

- When $a>b$, this interval means the set of all non-increasing functions $f(x)$ for which the range is exactly $[a, b]$. Since the function is non-increasing, its largest value is attained at $x=0$ and its smallest value is attained at $x=1$, hence $f(0)=a$ and $f(1)=b$.

In both cases, $f(0)=a$ and $f(1)=b$.

In this interpretation, + is interpreted as the set of all monotonic functions $h$ that can be represented as $h=f_{1}+f_{2}$, where $f_{1} \in\left[a_{1}, b_{1}\right]$ and $f_{2} \in\left[a_{2}, b_{2}\right]$. Since $f_{1} \in\left[a_{1}, b_{1}\right]$, we have $f_{1}(0)=a_{1}$ and $f_{1}(1)=b_{1}$. Similarly, we have $f_{2}(0)=a_{2}$ and $f_{2}(1)=b_{2}$, hence $h(0)=f_{1}(0)+f_{2}(0)=a_{1}+a_{2}$ and $h(1)=f_{1}(1)+f_{2}(1)=b_{1}+b_{2}$.

The function $h$ is monotonic, so, we have two possibilities:

- if $a_{1}+a_{2} \leq b_{1}+b_{2}$, this function is non-decreasing hence it belongs to $\left[a_{1}+a_{2}, b_{1}+b_{2}\right] \rightarrow$, i.e., to $\left[a_{1}+a_{2}, b_{1}+b_{2}\right]$;

- if $a_{1}+a_{2}>b_{1}+b_{2}$, this function is non-increasing hence it belongs to $\left[b_{1}+b_{2}, a_{1}+a_{2}\right] \leftarrow$, i.e., also to $\left[a_{1}+a_{2}, b_{1}+b_{2}\right]$.

In both cases, every function from $\left[a_{1}, b_{1}\right]+\left[a_{2}, b_{2}\right]$ belongs to the interval class $\left[a_{1}+a_{2}, b_{1}+b_{2}\right]$. To complete the proof, it is sufficient to observe that $\left[a_{1}+a_{2}, b_{1}+b_{2}\right]$ cannot belong to a smaller class, because it attains both endpoints as values.

In particular, we have $[2,3]+[1,0]=[3,3]$ and $[0,1]+[2,-2]=[2,-1]$. 


\section{What Did We Gain? Some Understanding}

We can produce similar results for operations with different interval classes. As a result, we get a uniform description of different interval operations. It is always a good idea to have a uniform explanation for several different things; but, as we will show, on top of that, we gain some deeper understanding of interval operations.

Example: interval arithmetic lacks some algebraic properties of operations with real numbers, e.g., in general, there is no distributivity:

$$
A \cdot(B+C) \neq A \cdot B+A \cdot C .
$$

The traditional explanation of this non-distributivity is that in interval arithmetic,

$$
A \cdot(B+C)=\{a \cdot(b+c) \mid a \in A, b \in B, c \in C\},
$$

while

$$
A \cdot B+A \cdot C=\left\{a \cdot b+a^{\prime} \cdot c \mid a \in A, a^{\prime} \in A, b \in B, c \in C\right\} .
$$

Our functional interpretation enables us to provide an additional (qualitative) explanation for this non-distributivity. For example, let us consider the interpretation from the previous section in which each operation $\odot$ is interpreted as $\odot \leftrightarrow$; in other words, the functions corresponding to all the intermediate results must be monotonic. Hence:

- In both sides, we compute, for every $x \in[0,1]$, the same value

$$
a(x) \cdot(b(x)+c(x)) \equiv a(x) \cdot b(x)+a(x) \cdot c(x) .
$$

- In the left-hand side, the intermediate results are $b+c$ and $a \cdot(b+c)$. This means that in defining this expression, we assume that the functions $b+c$ and $a \cdot(b+c)$ are monotonic.

- In the right-hand side, the intermediate results are $a \cdot b, a \cdot c$, and $a \cdot(b+c)$. This means that in defining this expression, we assume that the functions $a \cdot b, a \cdot c$, and $a \cdot(b+c)$ are monotonic.

These are two different monotonicity assumptions, so no wonder we sometimes get different results.

\section{What Next?}

In our definitions, as the domain of all the functions, we used the simplest possible case when we have only one variables, and we the simplest possible interval - the interval $[0,1]$. Natural questions:

- What is we use some other interval as a domain? one can easily see that the choice of the basic interval does not matter - all the results are valid for any basic interval.

- What if we use a multi-D domain $D \subseteq R^{n}$ ? In this case, we can define an interval as

$$
[\underline{a}, \bar{a}]_{F} \stackrel{\text { def }}{=}\{f \mid f \in C(D, R) \& f(D) \subseteq[\underline{a}, \bar{a}]\},
$$

where $C(D, R)$ denotes the class of all continuous functions $f: D \rightarrow R$. In 1-D case, monotonicity enabled us to consider, instead of the single original class (1), three different classes: 
- the original class (1) in which we impose no restrictions on how the function $f(x)$ depends on $x$;

- the class (3) in which we impose the restriction that the function $f(x)$ be non-decreasing in $x$, and

- the class (4) in which we impose the restriction that the function $f(x)$ be non-increasing in $x$.

In $n$-dimensional case, for each of $n$ variables $x_{i}$, we can impose one of the similar three restrictions. Specifically:

- first, we can consider all continuous functions irrespective of how they depend on $x_{i}$;

- second, we can impose the restriction that the function $f\left(x_{1}, \ldots, x_{i}, \ldots, x_{n}\right)$ be nondecreasing in $x_{i}$, and

- third, we can impose the restriction that the function $f\left(x_{1}, \ldots, x_{i}, \ldots, x_{n}\right)$ be nonincreasing in $x_{i}$.

Selecting one of these restrictions for each of the $n$ variables, we get $3^{n}$ different classes of functions. It would be interesting to determine what happens if we apply an arithmetic operation to two classes of different (or even same) type.

\section{Acknowledgments}

This work was supported in part by German Science Foundation, by NASA under cooperative agreement NCC5-209 and grant NCC2-1232, by Future Aerospace Science and Technology Program (FAST) Center for Structural Integrity of Aerospace Systems, effort sponsored by the Air Force Office of Scientific Research, Air Force Materiel Command, USAF, under grants numbers F4962095-1-0518 and F49620-00-1-0365, by NSF grants CDA-9522207, EAR-0112968, EAR-0225670, and 9710940 Mexico/Conacyt, by IEEE/ACM SC2001 and SC2002 Minority Serving Institutions Participation Grants, and by a research grant from Sandia National Laboratories as part of the Department of Energy Accelerated Strategic Computing Initiative (ASCI).

This research was partly done when J.W.v.G. was a Visiting Researcher at the University of Texas at El Paso. The authors are thankful to all the participants of SCAN'02, especially to S. Markov and S. Utyubaev, for valuable discussions, and to the anonymous referees for valuable suggestions.

\section{References}

[1] L. Jaulin, M. Kieffer, O. Didrit, and E. Walter, Applied Interval Analysis, with Examples in Parameter and State Estimation, Robust Control and Robotics, Springer-Verlag, London, 2001.

[2] E. Kaucher, Über metrische und algebraische Eigenschaften einiger beim numerischen Rechnen auftretender Raume, Ph.D. Dissertation, Universität Karlsruhe, 1973.

[3] E. Kaucher, "Algebraische Erweiterungen der Intervallrechnung unter Erhaltung der Ordnungs und Verbandstrukturen", Computing, Suppl. 1, 1977, pp. 65-79.

[4] E. Kaucher, "Über Eigenschaften und Anwendungsmöglichkeiten der erweiterten Intervallrechnung und des hyperbolische Fastkörpers über R", Computing, Suppl. 1, 1977, pp. 81-94. 
[5] E. Kaucher, "Interval Analysis in the Extended Interval Space IR", Computing, Suppl. 2, 1980, pp. 33-49.

[6] R. B. Kearfott, Rigorous Global Search: Continuous Problems, Kluwer, Dordrecht, 1996.

[7] R. B. Kearfott and V. Kreinovich (eds.), Applications of Interval Computations, Kluwer, Dordrecht, 1996.

[8] V. Kreinovich, A. Lakeyev, J. Rohn, and P. Kahl, Computational complexity and feasibility of data processing and interval computations, Kluwer, Dordrecht, 1997.

[9] L. Kupriyanova, "Inner Estimation of the United Solution Set of Interval Linear Algebraic System", Reliable Computing, 1995, Vol. 1, No. 1, pp. 15-31.

[10] S. M. Markov, "Some Interpolation Problems Involving Interval Data", Interval Computations, 1993, No. 3, pp. 164-182.

[11] S. M. Markov, "On Directed Interval Arithmetic and its Applications", Journal of Universal Computer Science, 1995, Vol. 1, No. 7, pp. 514-526.

[12] S. M. Markov, "On the Algebra of Intervals and Convex Bodies", Journal of Universal Computer Science, 1995, Vol. 4, No. 1, pp. 34-47.

[13] S. Markov, E. Popova, and Ch. Ullrich, "On the Solution of Linear Algebraic Equations Involving Interval Coefficients", in: S. Margenov, P. Vassilevski (eds.), Iterative Methods in Linear Algebra, II, IMACS Series in Computational and Applied Mathematics, 1996, Vol. 3, pp. 216-225.

[14] R. E. Moore, Methods and Applications of Interval Analysis, SIAM, Philadelphia, 1979.

[15] H.-J. Ortolf, "Eine Verallgemeinerung der Intervallarithmetik", Geselschaft für Mathematik und Datenverarbeitung (Bonn), 1969, Vol. 11, pp. 1-71.

[16] S. Shary, "Algebraic Approach to the Interval Linear Static Identification, Tolerance and Control Problems, or One More Application of Kaucher Arithmetic", Reliable Computing, 1996, Vol. 2, No. 1, pp. 1-32.

[17] S. Shary, "A New Approach to the Analysis of Static Systems under Interval Uncertainties", in: G. Alefeld and A. Frommer (eds.), Scientific Computing and Validated Numerics, Proceedings of SCAN-95, Akademie-Verlag, Berlin, 1996. 